\title{
Sperm competition in a fish with external fertilization: the contribution of sperm number, speed and length
}

\author{
J. A. STOLTZ \& B. D. NEFF \\ Department of Biology, University of Western Ontario, London, Ontario, Canada
}

\author{
Keywords: \\ bluegill; \\ fish; \\ mechanism; \\ raffle; \\ speed; \\ sperm competition; \\ sperm number.
}

\begin{abstract}
The role of sperm number and quality in male competitiveness was investigated using in vitro fertilization experiments with bluegill (Lepomis macrochirus). Bluegill males use one of three mating tactics: 'sneakers', which streak spawn; 'satellites', which mimic females; and 'parentals', which are territorial. The in vitro experiments mimicked natural spawning by incorporating these males' mean proximity to eggs and timing of sperm release. Using a maximum-likelihood algorithm, raffle equations were fit to paternity data, which revealed a strong effect of sperm number on male competitiveness. There was no difference in sperm flagellum length, curvilinear swim speed or path linearity among the three male mating types, and these traits did not explain any additional variation in male competitiveness. It was estimated that, given closer proximity to eggs, satellites need release only 0.34 times as many sperm as parentals to obtain equal paternity. Despite being farther from the eggs and releasing sperm about half a second after parentals, sneakers need only release 0.58 times as many sperm as parentals to obtain equal paternity. Thus, the increased competitiveness of sneakers' sperm must come from a component of sperm quality other than speed or length.
\end{abstract}

\section{Introduction}

Sperm competition occurs when the sperm from two or more males compete for the fertilization of a given set of eggs (Hildemann \& Wagner, 1954; Parker, 1970). Sperm competition is widespread, and occurs in a range of animal taxa including amphibians, birds, fishes, insects and mammals (Birkhead \& Møller, 1998). Sperm competition can lead to a wide range of behavioural, morphological and physiological adaptations that enhance the success of a male's sperm relative to a rival (Beatty et al., 1969; Parker, 1970; Smith, 1984). It is now well understood that males typically differ in their competitiveness during fertilization (Gage et al., 1995; Arnqvist \& Danielsson, 1999). However, the mechanisms that males use during sperm competition to increase their

Correspondence: B. D. Neff, Department of Biology, University of Western Ontario, London, Ontario N6A 5B7, Canada.

Tel.: 519850 2532; fax: 519661 3935;

e-mail: bneff@uwo.ca

Present address: J. A. Stoltz, Department of Life Sciences, University of Toronto at Scarborough, Scarborough, Ontario MlC 1A4, Canada

Both authors contributed equally to this work. probability of fertilization are just beginning to be understood (e.g. Birkhead et al., 1999; Evans et al., 2003; Gage \& Morrow, 2003; García-González \& Simmons, 2005).

In externally fertilizing fishes, sperm competition ranges from near complete mate monopolization to large breeding assemblages (Stockley et al., 1997). Taborsky (1998) postulated that success in sperm competition depends directly on a male's investment in gametes and semen. Specifically, during spawning, four components that can influence the probability of fertilization include: (1) proximity of males to eggs during ejaculation; (2) timing of sperm release and coordination with female egg release; (3) number of sperm released and (4) sperm traits such as swimming speed.

First, the proximity of males to females during sperm competition is important because males that ejaculate closer to females should give their sperm a competitive advantage in a race to fertilize the eggs. Furthermore, eggs and sperm may quickly diffuse, particularly when they are released in flowing or turbulent water (Petersen \& Warner, 1998; Petersen et al., 2001). Closer proximity of a male to a female during egg release has been demonstrated to increase a male's probability of 
fertilization during sperm competition in several species including brook trout, Salvelinus fontinalis (Blanchfield et al., 2003), Atlantic cod, Gadus morhua (Hutchings et al., 1999) and coho salmon, Oncorhynchus kisutch (Schröder, 1973).

Secondly, timing of sperm release is important because coordination with female egg release can increase the probability of sperm-egg collisions and ovarian fluids can modify the behaviour of sperm influencing the outcome of sperm competition. If a male releases sperm too early, then the concentration of sperm could diffuse and become too dilute (Levitan, 1998; Petersen \& Warner, 1998; Petersen et al., 2001) or the sperm could die or otherwise cease forward motion by the time a female releases her eggs. If a male releases sperm too late, then the eggs may be already fertilized. Hoysak \& Liley (2001) concluded that in sockeye salmon (Oncorhynchus nerka) a brief window exists during which eggs are available to be fertilized and that the proximity of males to females and timing of sperm release are critical factors determining success in sperm competition. In the Arctic charr (Salvelinus alpinus) the presence of ovarian fluid increases sperm longevity, swimming speed and linearity of sperm movement even though ovarian fluid is more viscous than freshwater (Turner \& Montgomerie, 2002). Cations such as $\mathrm{Mg}^{2+}$ and $\mathrm{Na}^{+}$in the ovarian fluid may increase motility and longevity of sperm (e.g. Linhart et al., 2002; Alavi et al., 2004).

Thirdly, sperm number is important for fertilization success when sperm competition follows a raffle-like process. In a raffle, each male's probability of fertilization is related to the number of sperm he contributes to the competition (Parker, 1990). For example, in bluegill (Lepomis macrochirus), in vitro fertilization trials that competed ejaculates from pairs of males showed that a male's paternity increased as his relative sperm number increased (Neff et al., 2003). However, another study on Atlantic salmon (Salmo salar) found no relationship between paternity and sperm number (Gage et al., 2004).

Fourthly, sperm traits such as swimming speed can be important during sperm competition (reviewed by Snook, 2005). Faster swimming sperm may be able to reach the egg first and thereby increase success during competition. For example, in Atlantic salmon, males with faster swimming sperm fertilized a greater proportion of eggs in two-male competition trials, and relative sperm swimming speed captured about half of the variation in fertilization success (Gage et al., 2004). Swim speed would be especially important if sperm also were able to home in on the egg (e.g. Turner \& Montgomerie, 2002).

The purpose of the present study was to examine mechanisms used during sperm competition by males that utilize different mating tactics in bluegill. Specifically, we incorporated information on proximity of males to females and timing of sperm release to assess the contribution of sperm number, length and speed to fertilization success.

\section{Methods}

\section{Study species}

Male bluegill have a discrete life history polymorphism called 'parental' and 'cuckolder' (Gross, 1979; Gross \& Charnov, 1980). In lake Opinicon (Ontario, Canada; $\left.44^{\circ} 34^{\prime} \mathrm{N}, 76^{\circ} 19^{\prime} \mathrm{W}\right)$, parentals delay sexual maturation until 7 years of age (Gross, 1982). During the spawning season parentals compete to construct nests in colonies (Dominey, 1981; Gross, 1982). After nest construction is completed females arrive at the colony as a group. Spawning involves a female entering a parental's nest and through a distinctive motion called a 'dip', the female releases a small batch of eggs at the utmost horizontal position of her dip (Gross, 1982). A female may dip hundreds or even thousands of times before leaving the parental's nest. Spawning lasts for a day, after which the females leave the colony and the parentals remain to provide sole care to the developing offspring. Cuckolders mature precociously at 2 years of age and behave as 'sneakers' (Gross, 1982). Sneakers hide in the debris at the edge of a parental's nest and streak into the nest during a female dip, stop momentarily to release sperm and typically are aggressively chased out by the parental. Sneakers are successful at entering the nest in about $17 \%$ of intrusion attempts (Gross, 1982), and when successful they fertilize a mean of $89 \%$ of the eggs (Fu et al., 2001). At around 4 years of age, cuckolders begin to mimic female morphology and behaviour and are referred to as 'satellites' (Gross, 1982). A parental will allow access of a satellite to his nest during spawning and may even court the satellite, suggesting that the parental typically cannot distinguish between a satellite and a female (Dominey, 1980; Gross, 1982; Neff \& Gross, 2001). During spawning, satellites typically position themselves between the female and parental and fertilize a mean of $67 \%$ of the eggs (Fu et al., 2001). Parentals compete with sneakers in about $7 \%$ of female dips and with satellites in about $3 \%$ of female dips; parentals rarely experience competition simultaneously from both types of cuckolder males (Neff et al., 2003).

Using underwater cameras, we have previously determined that when parentals and sneakers are in direct competition for a batch of eggs, a parental's urogenital pore is a mean of $23 \mathrm{~mm}$ (range among nests $=8$ $36 \mathrm{~mm}$ ) from that of the female's, whereas a sneaker's urogenital pore is a mean of $38 \mathrm{~mm}$ (range among nests $=24-48 \mathrm{~mm}$ ) from that of the female's (Stoltz $\delta$ Neff, 2006). Furthermore, sneakers release their sperm an estimated $0.46 \mathrm{~s}$ (range among nests $=0.31-0.97 \mathrm{~s}$ ) after parentals. Satellites release their sperm at the same time as parentals and are a mean of $17 \mathrm{~mm}$ (range among nests $=11-26 \mathrm{~mm}$ ) from a female's urogenital pore, whereas when a satellite is present, parentals are a mean of $64 \mathrm{~mm}$ (range among nests $=42-84 \mathrm{~mm}$ ) from the female (Stoltz \& Neff, 2006). 
All of the experiments in the current study were conducted during the May-July breeding seasons of 2004 and 2005 at the Queen's University Biological Station on Lake Opinicon. At the onset of spawning, males and females were collected from colonies and brought back to our field laboratory (near the site of collection). An egg or ejaculate sample was collected by applying gentle pressure on a fish's abdomen. Male types were differentiated based on behaviour (at the time of collection), size and coloration (Gross, 1982). Males were anaesthetized with clove oil immediately prior to ejaculate collection and only males with free-flowing milt were used. All egg and ejaculate samples were used within 2 min of collection. Care was taken to ensure that no water or urine contaminated or activated the ejaculate sample (which was verified by viewing sperm samples with a microscope).

\section{Sperm traits}

We measured three sperm traits as possible indexes of quality: curvilinear swim speed, path linearity and flagellum length. To measure the first two traits, a total of $5 \mu \mathrm{L}$ of freshly collected ejaculate was added to $750 \mu \mathrm{L}$ high osmolarity extender $(20 \mathrm{~mm}$ Tris, $2 \mathrm{~mm} \mathrm{KCl}$, $200 \mathrm{~mm} \mathrm{NaCl}, \mathrm{pH} 9.0$ ). About $5 \mu \mathrm{L}$ of this mixture was then placed onto an Improved Neubaur haemocytometer (Fisher Scientific, Ottawa, ON, Canada) and covered with a slip. The haemocytometer was then placed under a Motic Digital Microscope (model DMB 1-223; Upstate Technical Equipment Company, East Syracuse, NY, USA). The sample was focused at a total magnification of $100 \times$ and then activated by flooding the haemocytometer with lake water (at $20^{\circ} \mathrm{C}$ ). The sample was recorded for a minimum of $60 \mathrm{~s}$. The video camera recorded 30 frames per second.

We analysed the videos using the NIH IMAGE J program (v. 1.34; National Institutes of Health, Bethesda, MD, USA). At both 5 and $10 \mathrm{~s}$ post-sperm activation, we analysed 11 consecutive frames (i.e. one-third of a second). A single primary grid $(0.2 \times 0.2 \mathrm{~mm})$ was selected on the haemocytometer from which 10 sperm were selected and tracked. The position of the sperm was recorded in each consecutive frame and these data were used to calculate the curvilinear speed of the sperm as well as the path linearity. Curvilinear speed incorporates the specific path followed by the sperm and equals the total distance travelled divided by the time to travel that distance; path linearity varies between 0 and 1, where a 0 implies the sperm started and ended at the same location and a 1 implies the sperm moved in a straight line (see Kime et al., 2001). The two time intervals were selected to encompass the possible fertilization window for the eggs in nature and the speed and path linearity values from the two times were averaged into a single index of speed for each male.
To measure flagellum length, $1 \mu \mathrm{L}$ of the freshly collected ejaculate was added to $150 \mu \mathrm{L}$ of fixative $(3 \%$ glutaraldehyde in $0.1 \mathrm{~m}$ cacodylate buffer, $\mathrm{pH} 7.0$ ). The fixed sample was spread across an Improved Neubaur haemocytometer and covered with a slip. A photograph was then taken of the sample using the digital microscope. The uthscsa image tool (v. 3.0; University of Texas Health Science Centre, San Antonio, TX, USA) was used to trace the flagellum of 10 haphazardly selected sperm and to determine the length of the flagellum. For each sperm, the flagellum was traced three times and these measurements were averaged (the three measurements were highly repeatable: $r>0.9$ for pairs of data).

\section{Sperm competitiveness}

We tested ejaculate competitiveness of sneakers, satellites and parentals by using an experiment involving sperm number, distance from eggs during ejaculation and time difference between male ejaculations (for sneakers only). For each successive competition trial, we alternated which male type (cuckolder or parental) was used first to collect the ejaculate. Various volumes of ejaculate, ranging from 0.5 to $9.6 \mu \mathrm{L}$ from a sneaker or satellite and 0.5 or $1.0 \mu \mathrm{L}$ from a parental, were introduced above 30-100 eggs (freshly collected from a gravid female) from two separate pipettes. These volumes were selected to provide a range of sperm numbers for competing males. The pipettes were positioned to mimic the actual mean distances between the male and female urogenital pores as determined from previous video analysis (Stoltz \& Neff, 2006).

For the sneaker-parental trials the sperm from the sneaker was introduced shortly after that of the parental to correspond to the time difference calculated from the films. To determine our accuracy to simulate the time difference the experimenter trained using a timing device that was triggered in a similar manner to the operation of the pipettes. Based on the timing device, we determined that we could precisely repeat a time delay of $0.46 \mathrm{~s}$ $\left(\right.$ mean $=0.47 \mathrm{~s}$; range $=0.43-0.53 \mathrm{~s}$; one-sample $t_{19}=$ $1.51, P=0.15 ; 0.46 \mathrm{~s}$ was the estimated time delay for sneakers). For satellite-parental trials the sperm were introduced simultaneously.

Sperm density was calculated for each male after the in vitro sperm introduction by taking a sample of $3 \mu \mathrm{L}$ of the same milt sample used in the experiment. The $3 \mu \mathrm{L}$ were diluted 150-fold with water and a sample of this solution was placed on an Improved Neubaur haemocytometer. The sperm were counted in ten $0.2 \times 0.2 \mathrm{~mm}$ grids under a compound light microscope at $400 \times$ magnification. Sperm density was calculated by multiplying the mean sperm count per grid by the dilution factor and volume of liquid in each cell.

The fertilized eggs were maintained in $1 \mathrm{~L}$ jars with aeration and at ambient temperature on a natural light cycle for 7 days. Water changes (with lake water) were performed daily. At the end of the 7 days, all larvae were 
preserved in $95 \%$ ethanol. DNA was later isolated from the adults and a subset of larvae, and microsatellite DNA fingerprints were obtained by using multiplex genotyping (Colbourne et al., 1996; Neff et al., 2000). Up to four primer sets (locus: RB7, RB20, LMAR10, LMAR 14; published in DeWoody et al., 1998 and Schable et al., 2002) were simultaneously amplified, and all competing parental and cuckolder offspring were unambiguously identified by using exclusion methods (Chakraborty et al., 1988).

Based on the paternity data, we used Neff $\delta$ Wahl's (2004) maximum-likelihood algorithm to fit the following raffle equation:

$$
\frac{N_{\mathrm{c}}}{N_{\mathrm{c}}+N_{\mathrm{p}}}=\frac{S_{\mathrm{c}}^{t}}{S_{\mathrm{c}}^{t}+r S_{\mathrm{p}}^{t}} ;
$$

where $N_{\mathrm{c}}$ is the number of offspring sired by the cuckolder and $N_{\mathrm{p}}$ is the number of offspring sired by the parental, $S_{\mathrm{c}}$ and $S_{\mathrm{p}}$ are the numbers of sperm provided by the cuckolder and parental, $r$ is a measure of the loading given to the parental's sperm relative to the cuckolder's sperm and $t$ is a measure of the economy of scale to sperm number (i.e. a nonlinear effect of sperm number; see Parker, 1990; Neff \& Wahl, 2004). Neff $\delta$ Wahl's (2004) algorithm determines the most-likely value of $r$ and $t$ and whether or not these values differ significantly from the fair-raffle model $(r=1 ; t=1)$ and from the sperm independent model, in which case fertilization success is independent of sperm number $(t=0)$. Using the best-fit lines derived from eqn 1 , we calculated the coefficient of determinations for the two competition trails (Zar, 1999, p. 335).

By rearranging eqn 1, we next calculated the relative sperm number needed to explain equal fertilization success and the fertilization success observed in nature for competing males:

$$
\frac{S_{\mathrm{c}}}{S_{\mathrm{p}}}=\left(\frac{p r}{1-p}\right)^{\frac{1}{t}}
$$

where $p$ is the paternity of the sneaker or satellite as calculated from $N_{\mathrm{c}} /\left(N_{\mathrm{c}}+N_{\mathrm{p}}\right)$. The value of $p$ was set to either $50 \%$ or $89 \%$ for sneakers and either $50 \%$ or $67 \%$ for satellites; the latter two numbers in each pair represents the estimated fertilization success in nature (see Fu et al., 2001). To determine the sensitivity of the $S_{\mathrm{c}} / S_{\mathrm{p}}$ ratio for the fertilization success estimates in nature, we re-sampled the original data set with replacement 1000 times and recalculated $r$ and $t$ and $S_{\mathrm{c}} / S_{\mathrm{p}}$ (Manly, 1997). For the resulting distribution of $S_{c} / S_{\mathrm{p}}$ values we assessed the 5 th and 95 th percentiles.

\section{Statistical analyses}

A Kruskal-Wallis nonparametric test was used to compare curvilinear swim speed among the three male types because this variable deviated from a normal distribution for one of the male types. Analysis of variance (ANOvA) was used to compare path linearity or flagellum length among the three male types because these variables did not deviate from a normal distribution. A linear regression was used to assess the relationship between flagellum length and either speed or path linearity. A Spearman's correlation was also used to assess the relationship between each of the sperm traits and male competitiveness. This was done by taking the difference in each trait between competing males in the sperm number trials [i.e. (sneaker trait-parental trait) or (satellite trait-parental trait)] and correlating the difference with the residuals from the raffle equations. For the correlation analyses, data from all males were combined. We used Spearman's correlation because, although the residuals did not significantly differ from zero $(P>0.10$ for each) and where symmetrically distributed about a mean of zero, they did significantly differ from a normal distribution (Shapiro-Wilks, $P<0.001$ ). We did not measure all sperm traits in each field season, so sample sizes vary among the different analyses. All sperm traits were performed by a researcher who was blind to the male type. All statistics were performed using JMP (v. 4.0.4; SAS, Cary, NC, USA); mean values are reported \pm 1 SE.

\section{Results}

\section{Sperm traits}

Curvilinear speed was variable among sperm within ejaculates with a mean coefficient of variation of $35 \%$. Sperm length was less variable with a mean coefficient of variation of $8 \%$. However, the 10 sperm measured should provide precise mean estimates for each male; for example, the standard error relative to the mean for speed and length were $11 \%$ and $2.5 \%$ respectively. Across all males, the sperm curvilinear speed measurements at 5 and $10 \mathrm{~s}$ post-activation were highly correlated $(r=0.93, P<0.001, n=24)$. The path linearity index was also correlated at these two times $(r=0.70$, $P<0.001, n=24)$. The means of the 5 and $10 \mathrm{~s}$ values were not significantly different among the three male types for speed (Fig. la; parental: $150 \pm 12 \mu \mathrm{m} \mathrm{s}^{-1}$; sneaker: $137 \pm 13 \mu \mathrm{m} \mathrm{s}^{-1}$; satellite: $165 \pm 11 \mu \mathrm{m} \mathrm{s}^{-1}$; $\chi_{2}^{2}=3.10, P=0.21$ ) or path linearity (Fig. 1b; parental: $0.75 \pm 0.03$; sneaker: $0.76 \pm 0.03$; satellite: $0.78 \pm 0.03$; $\left.F_{2,21}=0.21, P=0.82\right)$. There was also no difference in the mean flagellum length across the three male types (Fig. 2; parental: $40.1 \pm 0.6 \mu \mathrm{m}$; sneaker: $40.4 \pm 0.6 \mu \mathrm{m}$; satellite: $\left.40.1 \pm 0.6 \mu \mathrm{m} ; F_{2,35}=0.10, P=0.91\right)$. Flagellum length was not related to either speed $\left(r^{2}=0.0064\right.$, $\beta=0.08, \quad P=0.72, n=24)$ or path linearity $\left(r^{2}=\right.$ $0.062, \beta=-0.25, P=0.23, n=24)$.

\section{Sperm competitiveness}

We conducted 84 competition trials involving either a sneaker and parental $(n=37)$ or a satellite and parental 

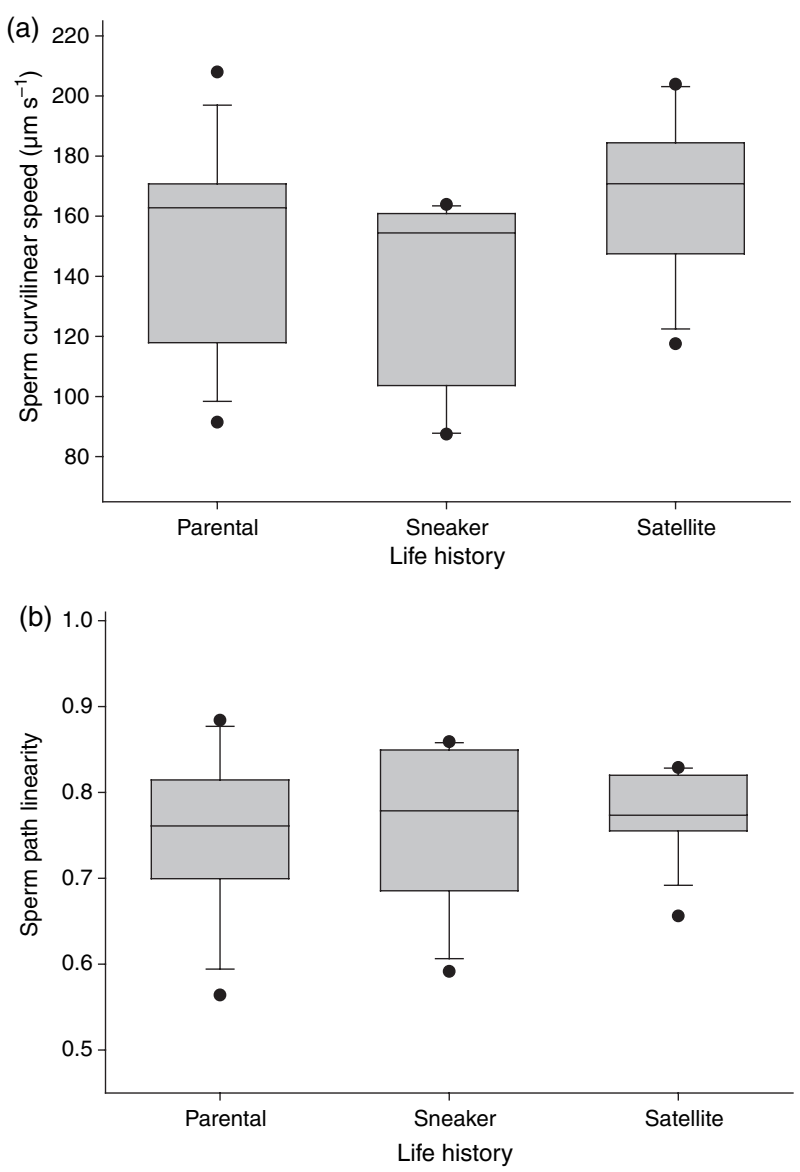

Fig. 1 Sperm traits for parental, sneaker and satellite male bluegill (Lepomis macrochirus). Data are (a) curvilinear speed and (b) path linearity and are represented by box plots that summarize the 10th, 25th, 50th, 75th and 90th percentiles. Dots denote data points that fall outside the 10th-90th percentile range. Sample sizes are 8, 7 and 9 for parental, sneaker and satellite respectively. There was no statistical difference in either measure among the three male mating tactics (see 'Results' for statistics). A path linearity value of 0 implies the sperm started and ended at the same location, whereas a value of 1 implies the sperm moved in a straight line.

$(n=47)$. For parentals, sperm density had a mean of $4.3 \times 10^{6}$ sperm $\mu \mathrm{L}^{-1} \quad$ (range $=0.9 \times 10^{6}-14.7 \times 10^{6}$ sperm $\mu \mathrm{L}^{-1}$ ), for sneakers, sperm density had a mean of $10.5 \times 10^{6}$ sperm $\mu \mathrm{L}^{-1} \quad$ (range $=5.0 \times 10^{6}-22.7 \times$ $10^{6}$ sperm $\left.\mu \mathrm{L}^{-1}\right)$, and for satellites, sperm density had a mean of $10.7 \times 10^{6}$ sperm $\mu \mathrm{L}^{-1} \quad$ (range $=0.8 \times 10^{6}$ $23.7 \times 10^{6}$ sperm $\mu \mathrm{L}^{-1}$ ).

In total, we determined the genotypes of 1632 offspring with a mean of 19 offspring per trial (range $=7-36$ offspring per trial). For the sneakerparental trials, the maximum-likelihood algorithm revealed that the sperm loading $r$ was 0.58 and the economy of scale $t$ was 1.00 (Fig. 3a). This model was nonlinear and did not conform to the fair-raffle because the $r$ parameter was significantly different from 1

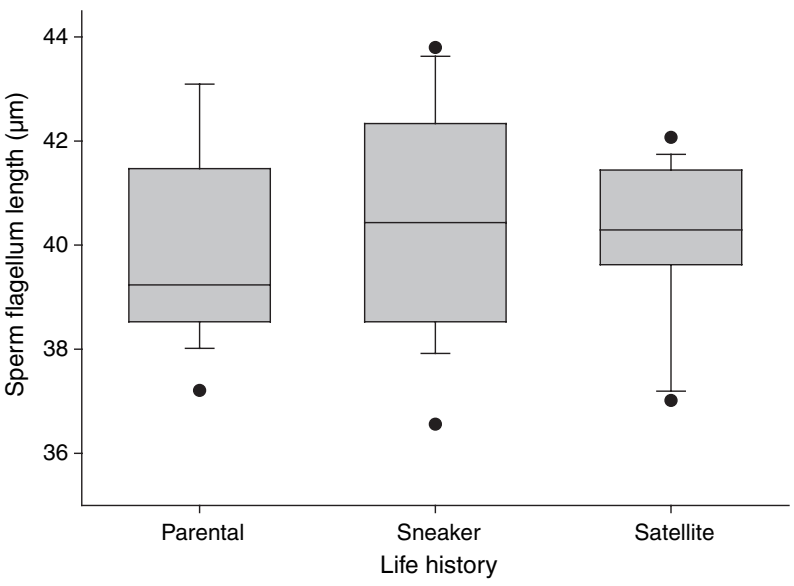

Fig. 2 Flagellum length of sperm from parental, sneaker, and satellite male bluegill (Lepomis macrochirus). Data are represented by box plots that summarize the 10th, 25th, 50th, 75th and 90th percentiles. Dots denote data points that fall outside the 10th-90th percentile range. Sample sizes are 13, 14 and 11 for parental, sneaker and satellite respectively. There was no statistical difference in flagellum length among the three male mating tactics (see 'Results' for statistics).

$(P<0.001)$. The equation of the line captured $75 \%$ of the variation (i.e. $r^{2}=0.75$ ). For the satellite-parental trials, the algorithm revealed that the sperm loading $r$ was 0.35 and the economy of scale $t$ was 0.95 (Fig. 3b). This model was similarly nonlinear and did not conform to the fair-raffle because the $r$ parameter was significantly different from $1 \quad(P<0.001)$. The equation of the line captured $51 \%$ of the variation (i.e. $r^{2}=0.51$ ). For both sets of trials the $t$ parameter was not significantly different than 1 ( $P>0.48$ for both), but $t$ was significantly different than 0 ( $P<0.001$ for both) and therefore we were able to reject the sperm independent model.

When we removed the effect of sperm number by using the residual paternity from the raffle equations, we found no relationship between males' competitiveness and the difference in males' sperm flagellum length $\left(r_{\mathrm{S}}=-0.20, P=0.49, n=14\right)$, curvilinear speed $\left(r_{\mathrm{S}}=\right.$ $-0.11, P=0.75, n=11)$, or path linearity $\left(r_{\mathrm{S}}=-0.34\right.$, $P=0.31, n=11)$. Sample sizes are smaller for these latter analyses because we did not always have sperm trait measurements for both males in a competition trial.

Using eqn 2 and setting $p$ to $50 \%$, we determined that despite the disadvantaged position, sneakers need to release only 0.58 times the number of sperm that parentals release to obtain equal fertilization success. For satellites, we determined that they need to release only 0.34 times the number of sperm that parentals release to obtain equal fertilization success. By instead setting $p$ to $89 \%$ for sneakers and $67 \%$ for satellites (the estimated fertilization success in nature; Fu et al., 2001), we determined that sneakers release an estimated 4.7 ( 5 th and 95th percentiles $=2.3-8.3$ ) times the number 

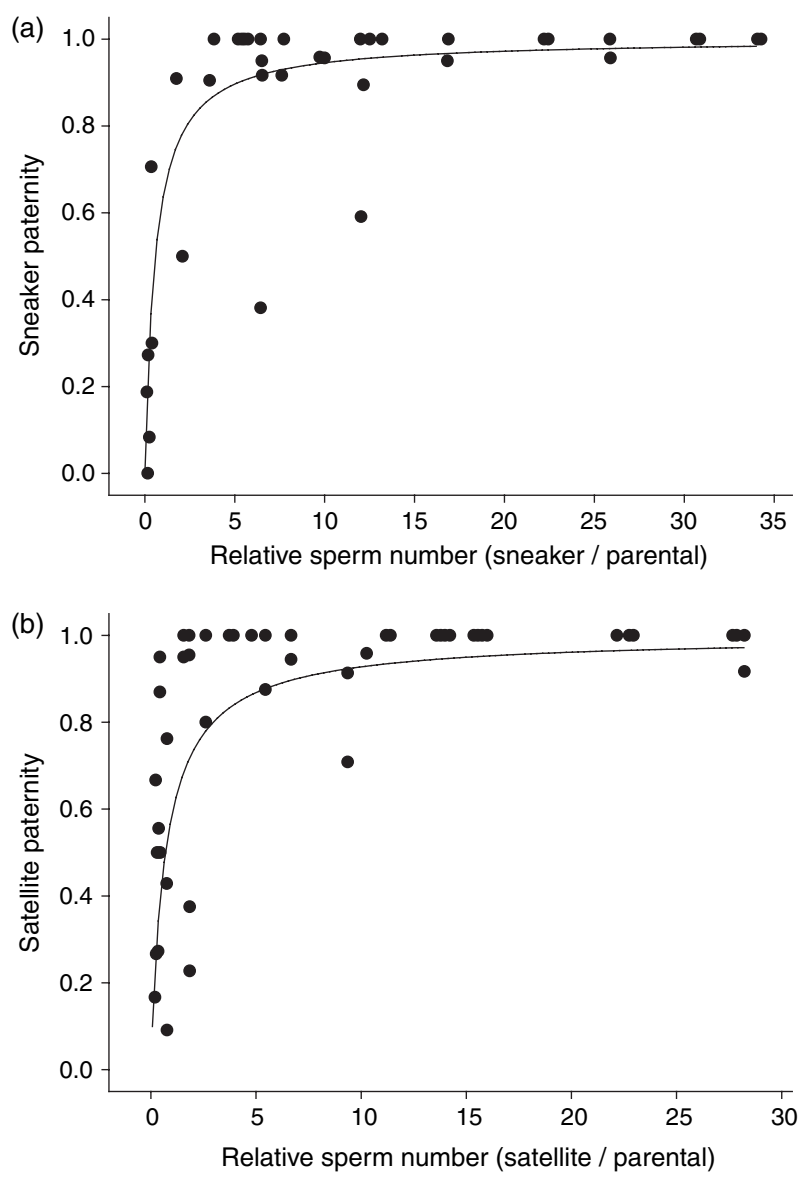

Fig. 3 The relationship between sperm number and male competitiveness in in vitro fertilization trials involving parental, sneaker and satellite male bluegill (Lepomis macrochirus). Competition trials involved (a) sneakers vs. parentals $(n=37)$ where the equation of the line is: sneaker paternity $=S_{\mathrm{c}}^{1.00} /\left[S_{\mathrm{c}}^{1.00}+0.58 S_{\mathrm{p}}^{1.00}\right]$ or $(\mathrm{b})$ satellites vs. parentals $(n=47)$ where the equation of the line is: satellite paternity $=S_{\mathrm{c}}^{0.95} /\left[S_{\mathrm{c}}^{0.95}+0.35 S_{\mathrm{p}}^{0.95}\right]$. The lines were fit using the raffle model of Neff $\&$ Wahl (2004).

of sperm relative to parentals and satellites release an estimated 0.70 (5th and 95th percentiles $=0.39-1.1$ ) times the number of sperm relative to parentals.

\section{Discussion}

Many fishes with external fertilization are characterized by sperm competition (Petersen \& Warner, 1998). Within these species, sperm traits such as swimming speed, number of sperm released, timing of sperm release and proximity of males to eggs all can influence a male's fertilization success (Hoysak \& Liley, 2001; Blanchfield et al., 2003; Neff et al., 2003; Gage et al., 2004). In this study, we examined the role of sperm number and quality in male competitiveness in the bluegill (L. macrochirus) using in vitro fertilization experiments that mimicked natural spawning by incorporating proximity and timing of sperm release.

We found that sperm competition in bluegill does not conform to a 'fair raffle', but instead represent a 'loaded raffle' whereby one male's sperm has a competitive advantage over another male's sperm (Parker, 1990). Based on eqn 1 , an ' $r$ ' parameter $<1$ indicates that parental sperm are competitively inferior (see Neff $\delta$ Wahl, 2004). For sneakers, even though they are in a disfavoured role based on proximity and timing of sperm release, our competition trials revealed that their sperm still have an advantage over parental sperm $(r=0.58)$. It is unlikely that this competitive advantage relates to differences in sperm flagellum length or swim speed because we found no difference in these traits between any of the three male types (also see Leach $\&$ Montgomerie, 2000; Burness et al., 2005), and there was no relationship between the difference in these traits between two competing males and their competitiveness. However, caution is warranted for the latter conclusion because our sample sizes were small for those particular analyses ( $n=11$ or 14$)$ and the large variation in sperm numbers used in the competition trials may have reduced statistical power to detect independent effects of the sperm traits. Two studies also report no difference in sperm longevity between sneakers and parentals (Leach \& Montgomerie, 2000; Burness et al., 2005), although we did not assess longevity here.

Alternatively, it is possible that ATP concentrations contribute to the difference in competitiveness because sperm from sneakers have higher ATP concentration than those from parentals (Burness et al., 2004, 2005). For satellites, the model revealed that their sperm similarly have an advantage over parental sperm $(r=$ $0.35)$, but this could relate simply to the proximity advantage of satellites (Stoltz \& Neff, 2006). Interestingly, we have previously shown that when equal volumes of milt are mixed from pairs of parentals and either sneakers or satellites and placed in direct contact with eggs, parental sperm appear more competitive than cuckolder sperm (Neff et al., 2003; also see Schulte-Hostedde $\delta$ Burness, 2005). In conjunction with the data presented here, which incorporate proximity of males to eggs, the results suggest that the race to the egg is paramount in understanding males' competitiveness; although some care is needed when interpreting the data of Neff et al. (2003) because they did not have actual sperm counts for their males.

There are differences in the mechanisms that sneakers and satellite use to out-compete rival parentals during sperm competition. Sneakers intrude on a spawning female and parental by streaking in from the periphery of the parental's nest. Previously, we have shown that sneakers gain access to eggs about half a second after parentals and end up 1.6 times farther from the eggs than parentals during ejaculation (Stoltz \& Neff, 2006). Nevertheless, sneakers are able to fertilize an average of 
$89 \%$ of the eggs (Fu et al., 2001). Here, we used the in vitro fertilization experiment to predict that sneakers on average must release about 4.7 times more sperm than parentals in order to fertilize $89 \%$ of the eggs. Conversely, satellites temporarily position themselves between the female and parental during spawning and end up closer to the female at only an average of $26 \%$ of the distance that parentals are to females when eggs are released (Stoltz \& Neff, 2006). Based on the in vitro fertilization experiment, we predict that satellites release only about $70 \%$ as many sperm as parentals to fertilize the $67 \%$ of the eggs that Fu et al. (2001) calculated. These calculations of relative sperm number are only estimates as our analysis utilized the mean proximity of males to females and did not incorporate the variance in these estimates. This approach could introduce error into our sperm number estimates if there is a nonlinear relationship between proximity and sperm competitiveness. Furthermore, we were unable to incorporate potential differences in mechanisms of ejaculation, such as differences in musculature, and the effect of water turbulence from male and female movements during spawning. Nevertheless, our analysis suggests that sneakers outcompete parentals in part by releasing more sperm, whereas satellites out-compete parentals in part by obtaining a superior position during spawning. To confirm the former, however, requires measuring the numbers of sperm released in nature.

How is it possible that sneakers are able to release nearly five times more sperm than parentals? Leach $\delta$ Montgomerie (2000) showed that the total volume of milt in a stripped ejaculate of a sneaker had a mean of $11.9 \mu \mathrm{L}$, whereas that for parentals was over five times greater at $63.4 \mu \mathrm{L}$. First, we found that the density of sperm in ejaculates of sneakers is greater at about 2.4 times the density of the ejaculates of parentals (= $\left.10.5 \times 10^{6} / 4.3 \times 10^{6}\right)$. Leach \& Montgomerie (2000) estimated the relative density of sperm in ejaculates of sneakers and parentals to be $1.4 \quad\left(=16.5 \times 10^{6} \%\right.$ $\left.11.5 \times 10^{6}\right)$. Secondly, because sneakers overall access only about $7 \%$ of spawning events (i.e. dips) and outnumber parentals 3: 1 (Neff et al., 2003), individual sneakers access only $2.3 \% \quad(=7 / 3)$ the number of spawning events that individual parentals access. Based on these spawning numbers and the sperm density estimates of Leach \& Montgomerie (2000) (the lower estimate of relative sperm number), we can estimate the relative numbers of sperm per spawning event (dip) available for sneakers and parentals. Sneakers have a total of $196 \times 10^{6}$ sperm $\quad\left(=11.9 \mu \mathrm{L} \times 16.5 \times 10^{6}\right.$ sperm $\left.\mu \mathrm{L}^{-1}\right)$, whereas parentals have a total of $729 \times 10^{6}$ sperm $\quad\left(=63.4 \mu \mathrm{L} \times 11.5 \times 10^{6}\right.$ sperm $\left.\mu \mathrm{L}^{-1}\right)$. Thus, parentals have 3.7 times the number of sperm that sneakers have. However, because individual parentals participate in 43 times $(=1 / 0.023)$ the number of spawning events that sneakers do, individual sneakers actually have about 11 times $(=43 / 3.7)$ the number of sperm available per spawning event (dip). This latter number suggests that sneakers could easily release our estimated 4.7 times more sperm than parentals during individual intrusions. These calculations, however, do not incorporate potential regeneration rates of sperm during the single day of spawning. We are currently investigating testes histology and sperm regeneration rates in bluegill.

The importance of sperm traits for male competitiveness appears to differ among externally fertilizing fishes. In Atlantic salmon, using in vitro fertilization experiments, Gage et al. (2004) showed that sperm number had no effect on fertilization success of pairs of competing males, whereas relative sperm swim speed captured a large portion of the variance in competitiveness. In bluegill, our data suggest the opposite relationship; i.e. number is more important than speed. It is possible that the difference in these two studies is attributable to variation in ecology between the species. Salmon typically spawn in flowing rivers, whereas the bluegill we studied spawn in relatively calm waters of the lake. Indeed, the experiment of Gage et al. (2004) was conducted in a funnel stream that mimicked the flowing water of natural rivers and our experiment was conducted in still water that mimicked conditions on the lake bottom. Flowing water may favour faster swimming sperm, whereas still water may select for more sperm. Differences in the effect of ovarian fluid on sperm traits (e.g. Turner \& Montgomerie, 2002) also may differ among species and deserves attention. To our knowledge the Atlantic salmon study by Gage et al. (2004) is the only other study of an externally fertilizing fish that uses controlled experiments to investigate the roles of sperm quality and number on males' competitiveness (for an example in an internal fertilizing fish, see Evans et al., 2003 and for examples from other taxa see Birkhead et al., 1999; Gage \& Morrow, 2003; García-González \& Simmons, 2005).

In conclusion, we found no statistical difference in flagellum length, curvilinear speed, or path linearity of sperm from male bluegill that utilize three different mating tactics. Competition trials revealed that sperm number strongly influences fertilization success, and independent of sperm number, sperm from sneakers and satellites have a competitive advantage over those from parentals. Although satellites are closer to the eggs than parentals, sneakers are more distant than parentals and therefore some other aspect of sperm quality must contribute to the increased competitiveness of sperm from sneakers.

\section{Acknowledgments}

Elizabeth Clare, Angela Dang, Tim Hain, Joy Marcus, Margaret Orlowski and Nick Sweet provided field and laboratory assistance. Tim Hain, Trevor Pitcher and four anonymous reviewers provided helpful comments on the 
manuscript. Parts of the work were conducted at the Queen's University Biological Station where Frank Phelan and Floyd Connors provided logistical assistance. The work was supported by an Ontario Graduate Scholarship to JAS, and the Natural Science and Engineering Research Council of Canada and a Premiere's Research Excellence Award (grants to BDN).

\section{References}

Alavi, S.M.H., Cosson, J., Karami, M., Amiri, B.M. \& Akhoundzadeh, M.A. 2004. Spermatozoa motility in the Persian sturgeon, Acipenser persicus: effects of $\mathrm{pH}$, dilution rate, ions and osmolality. Reproduction 128: 819-828.

Arnqvist, G. \& Danielsson, I. 1999. Copulatory behavior, genital morphology, and male fertilization success in water striders. Evolution 53: 147-156.

Beatty, R.A., Bennett, G.H., Hall, J.G., Hancock, J.L. \& Stewart, D.L. 1969. An experiment with heterospermic insemination in cattle. J. Reprod. Fert. 19: 491-502.

Birkhead, T.R. \& Møller, A.P. 1998. Sperm Competition and Sexual Selection. Academic Press, CA.

Birkhead, T.R., Martínez, J.G., Burke, T. \& Froman, D.P. 1999. Sperm mobility determines the outcome of sperm competition in the domestic fowl. Proc. Roy. Soc. Lond. (B) 266: 1759-1764.

Blanchfield, P.J., Ridgway, M.J. \& Wilson, C.C. 2003. Breeding success of male brook trout (Salvelinus fontinalis) in the wild. Mol. Ecol. 12: 2417-2428.

Burness, G., Casselman, S.J., Schulte-Hostedde, A.I., Moyes, C.D. \& Montgomerie, R. 2004. Sperm swimming speed and energetics vary with sperm competition risk in bluegill (Lepomis macrochirus). Behav. Ecol. Sociobiol. 56: 65-70.

Burness, G., Moyes, C.D. \& Montgomerie, R. 2005. Motility, ATP levels and metabolic enzyme activity of sperm from bluegill (Lepomis macrochirus). Comp. Biochem. Physiol. 140: 11-17.

Chakraborty, R., Meagher, T.R. \& Smouse, P.E. 1988. Parentage analysis with genetic markers in natural populations. I. The expected proportion of offspring with unambiguous paternity. Genetics 118: 527-536.

Colbourne, J.K, Neff, B.D., Wright, J.M. \& Gross, M.R. 1996. DNA fingerprinting of bluegill sunfish (Lepomis macrochirus) using $(\mathrm{GT})_{\mathrm{n}}$ microsatellites and its potential for assessment of mating success. Can. J. Fish. Aquat. Sci. 53: 342-349.

DeWoody, J.A., Fletcher, D.E., Fletcher, E.E., Wilkins, S.D., Nelson, W.S. \& Avise, J.C. 1998. Molecular genetic dissection of spawning, parentage, and reproductive tactics in a population of redbreast sunfish, Lepomis auritus. Evolution 52: 18021810.

Dominey, W.J. 1980. Female mimicry in male bluegill sunfish a genetic polymorphism? Nature 284: 546-548.

Dominey, W.J. 1981. Maintenance of female mimicry as a reproductive strategy in bluegill sunfish (Lepomis macrochirus). Environ. Biol. Fish. 6: 59-64.

Evans, J.P, Zane, L., Francescato, S. \& Pilastro, A. 2003. Directional postcopulatory sexual selection revealed by artificial insemination. Nature 421: 360-363.

Fu, P., Neff, B.D. \& Gross, M.R. 2001. Tactic-specific success in sperm competition. Proc. Roy. Soc. Lond. (B) 268: 1105-1112.

Gage, M.J.G. \& Morrow, E.H. 2003. Experimental evidence for the evolution of numerous, tiny sperm via sperm competition. Curr. Biol. 13: 754-757.
Gage, M.J.G., Stockley, P. \& Parker, G.A. 1995. Effects of alternative male mating strategies on characteristics of sperm production in the Atlantic salmon (Salmo salar): Theoretical and empirical investigations. Phil. Trans. Roy. Soc. Lond. (B) 350: 391-399.

Gage, M.J.G., Macfarlane, C.P., Yeates, S., Ward, R.G., Searle, J.B. \& Parker, G.A. 2004. Spermatozoa traits and sperm competition in Atlantic salmon: relative sperm velocity is the primary determinant of fertilization success. Curr. Biol. 14: 44-47.

García-González, F. \& Simmons, L.W. 2005. Sperm viability matters in insect sperm competition. Curr. Biol. 15: 271-275.

Gross, M.R. 1979. Cuckoldry in sunfishes (Lepomis: Centrarchidae). Can. J. Zool. 57: 1507-1509.

Gross, M.R. 1982. Sneakers, satellites and parentals: polymorphic mating strategies in North American sunfishes. $Z$. Tierpsychol. 60: 1-26.

Gross, M.R. \& Charnov, E.L. 1980. Alternative male life histories in bluegill sunfish. Proc. Natl. Acad. Sci. USA 77: 6937-6940.

Hildemann, W.H. \& Wagner, E.D. 1954. Intraspecific sperm competition in Lebistes reticulatus. Am. Nat. 88: 87-91.

Hoysak, D.J. \& Liley, N.R. 2001. Fertilization dynamics in sockeye salmon and a comparison of sperm from alternative male phenotypes. J. Fish Biol. 58: 1286-1300.

Hutchings, J.A., Bishop, T.D. \& McGregor-Shaw, C.R. 1999. Spawning behaviour of Atlantic cod, Gadus morhua: evidence of mate competition and mate choice in a broadcast spawner. Can. J. Fish. Aquat. Sci. 56: 97-104.

Kime, D.E., Van Look, K.J.W., McAllister, B.G., Huyskens, G., Rurangwa, E. \& Ollevier, F. 2001. Computer-assisted sperm analysis (CASA) as a tool for monitoring sperm quality in fish. Comp. Biochem. Physiol. Part C 130: 425-433.

Leach, B. \& Montgomerie, R. 2000. Sperm characteristics associated with different male reproductive tactics in bluegills (Lepomis macrochirus). Behav. Ecol. Sociobiol. 49: 31-37.

Levitan, D.R. 1998. Sperm limitation, gamete competition, and sexual selection in external fertilizers. In: Sperm Competition and Sexual Selection (T. R. Birkhead \& A. P. Møller, eds), pp. 175-217. Academic Press, CA.

Linhart, O., Cosson, J., Mims, S.D., Shelton, W.L. \& Rodina, M. 2002. Effects of ions on the motility of fresh and demembranated paddlefish (Polyodon spathula) spermatozoa. Reproduction 124: 713-719.

Manly, B.J.F. 1997. Randomization, Bootstrap and Monte Carlo Methods in Biology, 2nd edn. Chapman and Hall, London.

Neff, B.D. \& Gross, M.R. 2001. Dynamic adjustment of parental care in response to perceived paternity. Proc. Roy. Soc. Lond. (B) 268: 1559-1565.

Neff, B.D. \& Wahl, L.M. 2004. Mechanisms of sperm competition: testing the fair raffle. Evolution 58: 1846-1851.

Neff, B.D., Fu, P. \& Gross, M.R. 2000. Microsatellite multiplexing in fish. Trans. Am. Fish. Soc. 129: 584-593.

Neff, B.D., Fu, P. \& Gross, M.R. 2003. Sperm investment and alternative mating tactics in bluegill sunfish (Lepomis macrochirus). Behav. Ecol. 14: 634-641.

Parker, G.A. 1970. Sperm competition and its evolutionary consequences in the insects. Biol. Revol. Chamb. Phil. Soc. 45: 525-567.

Parker, G.A. 1990. Sperm competition games: raffles and roles. Proc. Roy. Soc. Lond. (B) 242: 120-126.

Petersen, C.W. \& Warner, R.R. 1998. Sperm competition in fishes. In: Sperm Competition and Sexual Selection (T. R. Birkhead \& A. P. Møller, eds), pp. 435-463. Academic Press, CA. 
Petersen, C.W., Warner, R.R., Shapiro, D.Y. \& Marconato, A. 2001. Components of fertilization success in the bluehead wrasse, Thalassoma bifasciatum. Behav. Ecol. 2: 237-245.

Schable, N.A., Fischer, R.U. \& Glenn, T.C. 2002. Tetranucleotide microsatellite DNA loci from the dollar sunfish (Lepomis marginatus). Mol. Ecol. Not. 2: 509-511.

Schröder, S.L. 1973. Effects of density on the spawning success of chum salmon in an artificial spawning channel. Master thesis, University of Washington, Seattle.

Schulte-Hostedde, A.I. \& Burness, G. 2005. Fertilization dynamics of sperm from different male mating tactics in bluegill (Lepomis macrochirus). Can. J. Zool. 83: 1638-1642.

Smith, R.L. 1984. Sperm Competition and the Evolution of Animal Mating Systems. Academic Press, London.

Snook, R.R. 2005. Sperm in competition: not playing by the numbers. Trend. Ecol. Evol. 20: 46-53.
Stockley, P., Gage, M., Parker, G. \& Møller, A. 1997. Sperm competition in fishes: The evolution of testis size and ejaculate characteristics. Am. Nat. 149: 933-954.

Stoltz, J.A. \& Neff, B.D. 2006. Male size and mating tactic influence proximity to females during sperm competition in bluegill sunfish. Behav. Ecol. Sociobiol., 59: 81 1-818.

Taborsky, M. 1998. Sperm competition in fish: 'Bourgeois' males and parasitic spawning. Trend. Ecol. Evol. 13: 222-227.

Turner, E. \& Montgomerie, R. 2002. Ovarian fluid enhances sperm movement in Arctic charr. J. Fish Biol. 60: 1570-1579. Zar, J.H. 1999. Biostatistical Analysis, 4th edn. Prentice Hall, NJ.

Received 17 December 2005; revised 27 April 2006; accepted 9 May 2006 